\title{
Hydroelastic wake on a thin elastic sheet floating on water
}

\author{
Jean-Christophe Ono-dit-Biot, ${ }^{1}$ Miguel Trejo, ${ }^{2}$ Elsie Loukiantcheko, ${ }^{1}$ \\ Max Lauch, ${ }^{1}$ Elie Raphaël, ${ }^{2}$ Kari Dalnoki-Veress, ${ }^{1,2}$ and Thomas Salez ${ }^{3,4, *}$ \\ ${ }^{1}$ Department of Physics and Astronomy, McMaster University, \\ 1280 Main Street West, Hamilton, Ontario, L8S 4M1, Canada. \\ ${ }^{2}$ UMR CNRS Gulliver 7083, ESPCI Paris, PSL Research University, 75005 Paris, France. \\ ${ }^{3}$ Univ. Bordeaux, CNRS, LOMA, UMR 5798, F-33405 Talence, France. \\ ${ }^{4}$ Global Station for Soft Matter, Global Institution for Collaborative Research and Education, \\ Hokkaido University, Sapporo, Hokkaido 060-0808, Japan.
}

(Dated: June 4, 2019)

\begin{abstract}
We investigate the hydroelastic waves created by a perturbation moving at constant speed along a thin elastic sheet floating at the surface of deep water. Using a high-resolution cross-correlation imaging technique, we characterize the waves as a function of the perturbation speed, for different sheet thicknesses. The general theoretical expression for the dispersion relation of hydroelastic waves includes three components: gravity, bending and tension. The bending modulus and the tension in the sheet are independently measured. The experiments represent a direct test of the theory where all components, bending, stretching and gravity, cannot be neglected. Excellent agreement is found between the experimental data and the theoretical expression.
\end{abstract}

\section{INTRODUCTION}

In 1963, Richard Feynman described water waves in his famous Lectures on Physics [1] as "the worst possible example [of waves], because they are in no respect like sound and light; they have all the complications that waves can have". Several decades later, some questions remain unanswered and the study of water waves continues to be an area of great interest. For example, Kelvin's gravity wake behind a ship [2] still stimulates fundamental questions [3, 4]. Surface tension of the liquid-air interface also influences the wave propagation, resulting in gravito-capillary waves [58]. Unlike the gravity wake, the capillary waves appear ahead of the perturbation [6]. This is particularly relevant for the locomotion of insects [9-13], as well as for nanorheological applications involving e.g. atomic-force microscopy probes moving along thin viscous samples [14-16].

Other waves of interest are the ones that propagate on elastic plates and membranes. Their properties are dictated by both the bending and stretching rigidities of the material [17]. Floating such an elastic sheet on a liquid further leads to the coupling of the elastic waves to hydrodynamics. The resulting hydroelastic waves are of particular interest, as elastic sheets surrounded by fluids are ubiquitous in nature. Examples can be found in fluid mechanics [18, 19], geophysics [20-23], and biophysics [24]. Hydroelastic waves are also relevant to practical applications in civil engineering [25, 26], as well as in energy harvesting through piezoelectric flags [27] and control of energy radiation by trucks moving on ice sheets [28]. Interestingly, the propagation of such waves can be finely controlled in an optical-like fashion by using model thin sheets with heterogeneous elastic properties [29]. Different properties of these waves, such as the wave resistance or non-linear effects, have been further studied theoretically [30,31], including the overdamped limit of lubrication settings where viscosity dominates over fluid inertia [32-38]. The dispersion relation in the inertial case was analytically derived and found to depend on three components: gravity, bending and stretching [39]. A few experimental studies developed in different contexts have studied the limiting cases where only bending and stretching $[40,41]$, or gravity and bending $[20,21]$, contribute.

In this article, we study the hydroelastic waves created by a perturbation moving at constant speed relative to an elastic sheet floating on deep water. The waves are imaged using a high-resolution optical method. By using elastic sheets with different thicknesses, the bending modulus of the sheet is varied over more than two orders of magnitude. We find excellent agreement between experimental data and the general theoretical dispersion relation, accounting for the three different contributions: gravity, bending, and tension.

\footnotetext{
* thomas.salez@u-bordeaux.fr
} 


\section{EXPERIMENTAL METHODS}

A transparent annular tank (outer radius $R_{\text {out }}=50 \mathrm{~cm}$, inner radius $R_{\text {in }}=30 \mathrm{~cm}$ ) is filled with water to a depth of about $16 \mathrm{~cm}$, as shown in Fig. 1. Thin elastic sheets of Elastosil ${ }^{\circledR}$ Film 2030 (Wacker Chemie AG) with nominal thicknesses $h$ of 50,100,200, 250 and $350 \mu \mathrm{m}$, and lateral dimensions of $20 \mathrm{~cm} \times 16 \mathrm{~cm}$ are floated onto the surface of water. Thin rigid plastic supports $(18 \mathrm{~cm} \times 1 \mathrm{~cm} \times 0.1 \mathrm{~cm})$ are placed atop the radial edges of the elastic sheet (shown in black in Fig. 1) to ensure the sheets do not crumple.

(a)

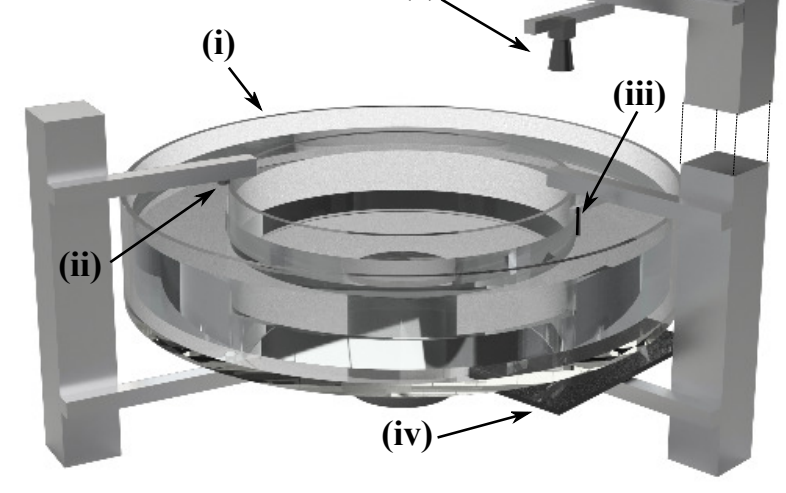

(b)

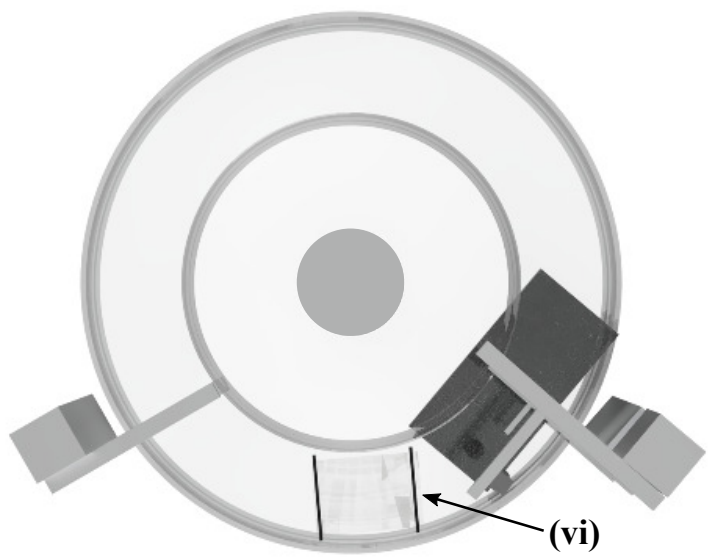

FIG. 1. (a) 3D schematics of the experimental setup. (i) Rotating transparent annular tank (outer radius $R_{\text {out }}=50 \mathrm{~cm}$, inner radius $R_{\text {in }}=30 \mathrm{~cm}$ ) filled with water to a depth of about $16 \mathrm{~cm}$, and with an elastic sheet floating atop. (ii) Infrared beam-breaking setup to measure the angular speed $\Omega$ of the elastic sheet. (iii) A pipette perturbs the surface by blowing air normal to to the surface, causing waves to form. (iv) Light sheet and dot pattern used to characterize the waves using the Schlieren method [42]. (v) A camera is placed $\sim 2 \mathrm{~m}$ above the tank to image the dot pattern. (b) Top-view schematics of the experimental setup. The elastic sheet (vi) only covers a small portion of the water surface. Plastic beams are placed along the radial edges of the elastic sheet as shown in black.

We experimentally verify that adding the supports does not introduce an anisotropic tension in the sheet, by ensuring that the deformation induced by ball bearings placed atop the sheet is axially symmetric (see Appendix). The tank is rotated at constant angular speed $\Omega$, ranging from 0 to $2.5 \mathrm{rad} . \mathrm{s}^{-1}$, causing the water to flow and the sheet to move. We take advantage of the opaque plastic supports to measure the angular speed of the sheet, using an infrared beam-breaking technique. Because of inertia, both the sheet and water do not follow the tank's speed instantaneously. Hence, all experiments are performed only once the speed of the sheet is constant and equal to the speed of the tank.

A glass capillary (World Precision Instruments, USA) is pulled to a diameter of about $100 \mu \mathrm{m}$ at one end with a pipette puller (Narishige, Japan), and used to blow air normal to the surface of the sheet (Fig. 1(iii)). The pipette is placed in the middle of the tank, i.e. at a radius $R_{\mathrm{p}}=40 \mathrm{~cm}$ from the center. The air jet acts as a perturbation moving at speed $v=\Omega R_{\mathrm{p}}$ in the reference frame of the elastic sheet, which generates hydroelastic waves. The latter is imaged using a synthetic Schlieren method [42] involving a random dot pattern refracted by the surface topography. The dot pattern is generated using Matlab [42,43] and printed onto a transparency film. Light shines through the dot pattern (Fig. 1(iv)) and the waves, before being collected by a camera above the tank (Fig. 1(v)). The Schlieren method consists in measuring the apparent displacement of the dots due to light refraction by the waves. The displacement is measured relative to a reference image of the unperturbed surface (i.e. no air jet) moving at angular speed $\Omega$ - which ensures that the collected information is only due to the waves. This measurement is performed using an open-source digital 2D image-correlation algorithm (Ncorr, Matlab) [44]. From the displacement of the dots, one can access the slope of the surface and thus the surface topography [42].

\section{RESULTS AND DISCUSSION}

\section{A. Measurement of the wavelength}

Figures 2(a,b) show a typical vectorial displacement field. The air jet creates a localized perturbation in the sheet, as evidenced by the large magnitude of the displacement field therein. Ahead of the perturbation, in the reference 
frame of the elastic sheet, the upstream wave pattern of the hydroelastic wave appears clearly, with a dominant centimetric - wavelength $\lambda$. Note that the displacement data plotted in Fig. 2 is directly related to the slope of the interface through Snell-Descartes' law, and that the amplitude of the waves is at least an order of magnitude smaller than the wavelength. As the hydroelastic waves propagate along the $y$-direction, the projection of the displacement along that direction provides the strongest signal for analysis. Figures $2(\mathrm{c}, \mathrm{d})$ thus focus only on the $y$-component $d$ of the displacement field.
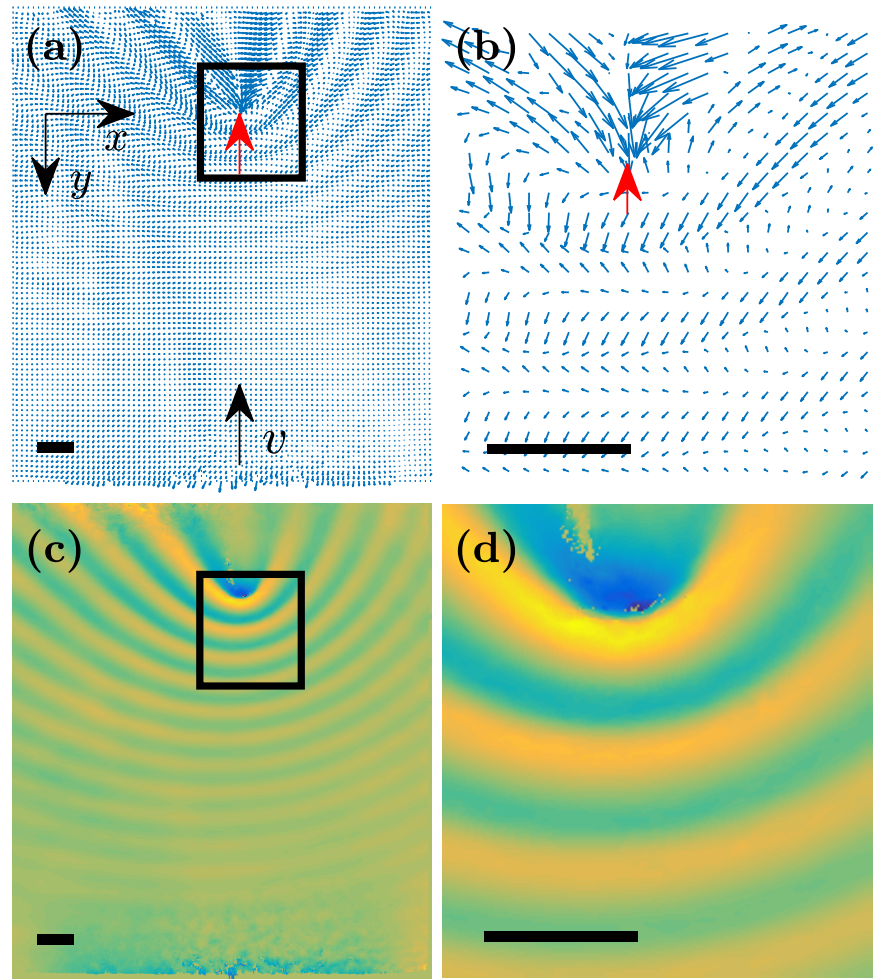

FIG. 2. (a) Raw 2D dot-displacement data measured with Ncorr [44], for an elastic sheet of nominal thickness $h \approx 200 \mu \mathrm{m}$ moved at speed $v=0.9 \mathrm{~m} . \mathrm{s}^{-1}$. The displacement vectors are only shown every 10 pixels for clarity. The bottom arrow indicates the speed of the sheet, with respect to the stationary air jet whose position is indicated by the red arrow. (b) Zoom around the perturbation, corresponding to the black box in (a). (c) The $y$-component $d$ of the displacement field. Warm colours (green to orange) correspond to positive displacements, while cold colours (green to blue) correspond to negative ones. (d) Zoom around the perturbation, corresponding to the black box in (c). All scale bars correspond to $1 \mathrm{~cm}$.

To characterize experimentally the dispersion relation of the hydroelastic wave, the wavelength $\lambda$ is measured as a function of the speed $v$. Figures 3(a-c) show the $y$-component $d$ of the displacement field, for various speeds. The wave is slightly tilted and not symmetric about the $y$-axis, because of the centrifugal force and the surface of water assuming a parabolic profile when rotated. This distortion is avoided in the wavelength measurement by analyzing the displacement field normal to the wave front, as shown in Fig. 3(a,d). We observe both the wavelength and the displacement magnitude to decrease as the speed increases. The general shape of the wave is consistent with a sinusoid with an exponential decay envelope (see Appendix). However, near the source of the perturbation, where the amplitude of the wave is the largest, the shape deviates from this description. The deviation may be ascribed to a loss of resolution for steep slopes and non-uniform strains in the elastic sheet near the source.

\section{B. Theoretical dispersion relation}

In order to quantify further and rationalize these observations, we now introduce the relevant theoretical framework which follows that of Schulkes and co-workers [39]. The mechanical system we consider is the thin elastic sheet in its reference frame. The out-of-plane displacement field $z=\zeta(\boldsymbol{r}, t)$ with respect to its flat horizontal state $z=0$ satisfies a simplified version of the Föppl-von Kármán equation [17]:

$$
B \nabla_{\boldsymbol{r}}^{4} \zeta-\sigma \nabla_{\boldsymbol{r}}^{2} \zeta+\rho_{\mathrm{e}} h \partial_{t t} \zeta=P+P_{\mathrm{ext}},
$$




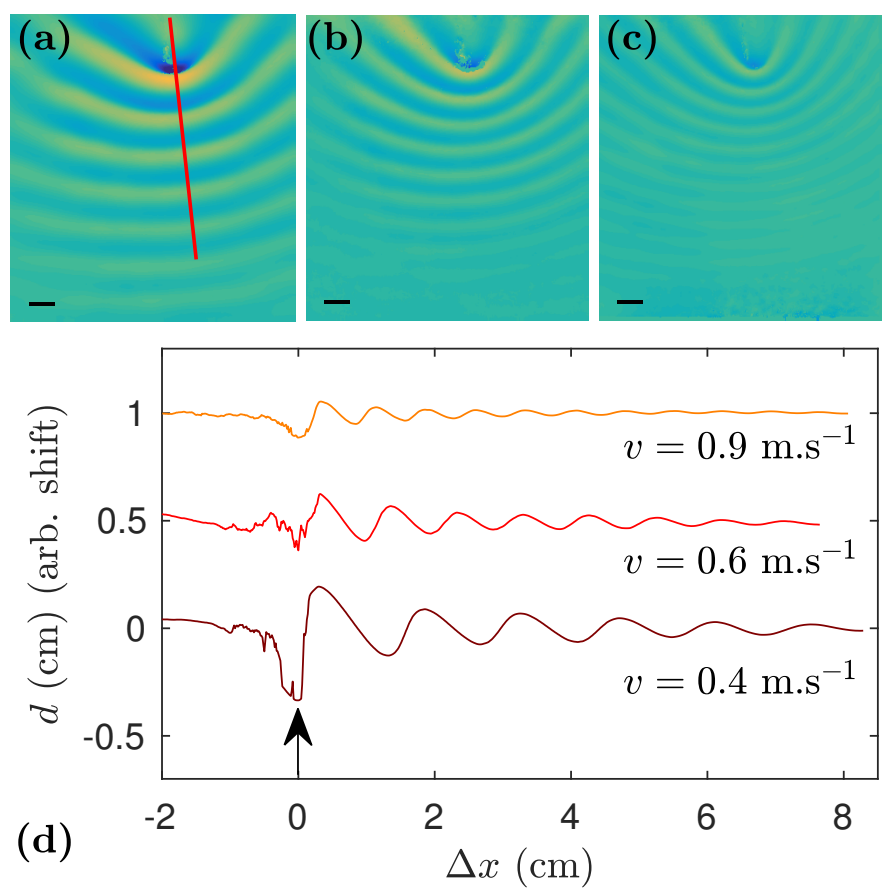

FIG. 3. (a) - (c) $y$-component $d$ of the displacement field (see Fig. 2), for speeds $v=0.4,0.6$, and 0.9 m.s ${ }^{-1}$, respectively, on an elastic sheet of nominal thickness $h \approx 200 \mu \mathrm{m}$. All scale bars correspond to $1 \mathrm{~cm}$. (d) $y$-component $d$ of the displacement field normal to the wave front (see red line in (a)), as a function of the distance $\Delta x$ from the perturbation, for the three speeds as indicated (shifted vertically for clarity). The position of the air-jet perturbation is indicated by the black arrow.

along the $2 \mathrm{D}$ horizontal space coordinate $\boldsymbol{r}=(x, y)$, and time $t$, where $\nabla_{\boldsymbol{r}}$ is the nabla operator in $2 \mathrm{D}, \partial_{t t}$ is the second partial derivative with respect to time, $B$ is the bending stiffness of the sheet, $\sigma$ represents the tension in the sheet, and $\rho_{\mathrm{e}}$ is the sheet's density. The first and second terms respectively account for bending and tension of the sheet, while the third term accounts for the solid inertia. The elastic sheet is further subjected to two external forces: the excess hydrodynamic pressure $P(\boldsymbol{r}, t)$ (with respect to the atmospheric one) exerted on the sheet by the water flow under gravity, and the driving pressure $P_{\text {ext }}(x, y-v t)$ modelling the perturbation by the air jet translating at constant speed $v$ along $y$. Note that we treat the motion as rectilinear, and that in particular we neglect the inertial effects due to rotation in view of the large radius of the tank and the moderate speed.

The water contribution, $P(\boldsymbol{r}, t)$, is calculated by assuming an incompressible and irrotational flow of an inviscid fluid, in a semi-infinite half space located at $z<\zeta(\boldsymbol{r}, t)$. In this context, the fluid velocity field can be written as $\nabla \varphi$, where $\varphi(\boldsymbol{r}, z, t)$ is a potential that vanishes in the far field and that satisfies Laplace's equation [45]: $\nabla^{2} \varphi=\left(\nabla_{\boldsymbol{r}}^{2}+\partial_{z}^{2}\right) \varphi=0$. At lowest order in the flow (i.e. for small-amplitude hydroelastic waves), the linearized Bernoulli equation for unsteady potential flows provides the excess hydrodynamic pressure exerted on the sheet: $P=-\rho \partial \varphi /\left.\partial t\right|_{z=0}-\rho g \zeta$, with $\rho$ the liquid density and $g$ the acceleration due to gravity.

To obtain the dispersion relation, one substitutes the expression for $P$ into Eq. (1) in the absence of driving $\left(P_{\text {ext }}=0\right)$, and invokes the kinematic condition $\partial \varphi /\left.\partial z\right|_{z=0}=\partial \zeta / \partial t$ at the water-sheet interface. Finally, the solid inertia can be neglected for films that are thin enough in comparison to the wavelength of the wave [39]. Considering a plane wave $\varphi \propto \exp [i(k y-\omega t-i k z)]$ satisfying Laplace's equation, with angular wavenumber $k$ and angular frequency $\omega(k)$, yields [39]:

$$
\omega=\sqrt{\frac{B k^{5}}{\rho}+\frac{\sigma k^{3}}{\rho}+g k} .
$$

We now consider the waves created by the driving perturbation $P_{\text {ext }}$ traveling at constant speed $v$ along $y$. In the comoving frame of the perturbation, the angular frequency $\omega^{\prime}$ of a plane-wave component of the waves is shifted by the Doppler effect, and thus reads $\omega^{\prime}=\omega-k v$. Furthermore, since in that comoving frame the wave is stationary, $\omega^{\prime}=0$ is a necessary condition. Using Eq. (2), one thus obtains the central relation connecting the angular wavenumber $k$ 
and the perturbation speed $v$, for a hydroelastic wave on deep water:

$$
v=\sqrt{\frac{B k^{3}}{\rho}+\frac{\sigma k}{\rho}+\frac{g}{k}} .
$$

An extensive analysis of this relation, similar to the one performed for the gravito-capillary case [7], reveals the main features of the present waves (see also Fig. 4). First, below a certain minimal speed $v^{*}$ (see Appendix) wave propagation is impossible. Secondly, at a given speed $v>v^{*}$ there are two possible values for the observed wavelength: i) the smallest value corresponds to a group velocity that is higher than the perturbation speed $v$, and therefore the waves propagate upstream of the perturbation. This is the situation studied in the present work (see Figs. 2 and 3), which is dominated by bending and tension at sufficiently large speed; ii) the largest value corresponds to a group velocity that is lower than the perturbation speed $v$, and therefore the waves propagate downstream of the perturbation. This situation corresponds to Kelvin's classical wake [2], which is dominated by gravity at sufficiently large speed.

\section{Experimental verification of the dispersion relation}

The values of the bending modulus $B$ and the tension $\sigma$ are measured independently. The bending modulus $B=E h^{3} /\left[12\left(1-\nu^{2}\right)\right]$ depends on three parameters: i) the Young's modulus $E=1.11 \pm 0.06 \mathrm{MPa}$ of Elastosil ${ }^{\circledR}$ Film 2030, measured using the stress-strain curve; ii) the sheet thickness $h$, depending on the sample and measured through optical microscopy; iii) the Poisson's ratio $\nu=0.5$, assuming that Elastosil ${ }^{\circledR}$ Film 2030 is an incompressible elastomer. The values of $h(\mu \mathrm{m})$ and $B(\mathrm{~N} . \mathrm{m})$ for our five different samples are found to be: $(h, B)=\{[51 \pm 1,(1.6 \pm$ $\left.\left.0.1) \times 10^{-8}\right],\left[104 \pm 2,(1.4 \pm 0.1) \times 10^{-7}\right],\left[213 \pm 7,(1.2 \pm 0.1) \times 10^{-6}\right],\left[258 \pm 2,(2.1 \pm 0.1) \times 10^{-6}\right],\left[362 \pm 3,(5.9 \pm 0.3) \times 10^{-6}\right]\right\}$. We note that the $362 \mu \mathrm{m}$ film was obtained by stacking two films with nominal thicknesses of $250 \mu \mathrm{m}$ and $100 \mu \mathrm{m}$. Besides, as the sheet is freely floating on water, the tension in the sheet is equal to the water-air surface tension: $\sigma=\gamma$. The latter is measured to be $\gamma=50 \pm 10 \mathrm{mN} \cdot \mathrm{m}^{-1}$, as in [29], from two different techniques (see Appendix): i) using a Wilhelmy-plate setup; ii) characterizing the dispersion relation of gravito-capillary waves on water. The low value of $\gamma$ and the large uncertainty are attributed to the fact that the tank is filled with an important volume of tap water, and thus subject to contamination by surfactants. We also note that recent works have shown that wrinkles can develop when a freely floating film is indented [46, 47]. In such a case, the tension in the film is no longer uniform and is in particular not equal to the water-air surface tension. In contrast, in our work, the typical stress introduced by the perturbation is of order $\sim A^{2} / \lambda^{2} \ll 1$ (as estimated for a triangular indentation of sizes $A$ and $\lambda$ ), with $A$ the amplitude of the wave, and is negligible in comparison to the water-air surface tension.

As shown in Fig. 4 (dashed lines), using the above measured values of $B$ and $\sigma$, one can predict the evolution of the angular wavenumber $k$ as a function of the perturbation speed $v$. The uncertainties in $B$ and $\sigma$ are taken into account through two limiting predictions and an interval in between. Note that the uncertainty on $\sigma$ accounts for most of the spread between the two limiting predictions. Finally, as expected from Eq. (3), all the theoretical curves for different $h$ (and thus $B$ ) collapse onto Kelvin's gravity-dominated branch [2], at both large $v$ and small $k$.

The wavelength $\lambda$ (see Fig. 3), or equivalently the angular wavenumber $k=2 \pi / \lambda$, is measured by fitting the experimental profile to a sinusoid with a decay envelope as a function of $v$ (see Appendix for more details) or by measuring the peak-to-peak distance. The results for the five different sheet thicknesses $h$ are shown in Fig. 4 (data points). We find excellent agreement between the experimental data and the theoretical prediction - that includes bending, tension, and gravity - with no adjustable parameter. The relative contributions of the three terms in Eq. (3) are discussed in details in the next section. The experimental data points for the two thinnest sheets seem to be in slightly better agreement with the upper prediction at low speed, and with the lower prediction at high speed. This observation could perhaps be related to a slight, but not quantifiable, increase in the sheet tension due to the increase in curvature of the water-air interface. Another interesting feature of Fig. 4 is that the difference between the upper and lower predictions decreases as the thickness $h$ of the sheet is increased. Indeed, the relative contribution of bending to Eq. (3) increases, and the difference between both predictions, which is mainly due to the uncertainty in tension, decreases. Note that Kelvin's classical gravity-dominated branch [2] corresponds to: i) a wake propagating behind the perturbation; ii) a wavelength that would almost reach the meter range in our experiments, which is not attainable with the current setup.

\section{Relative contributions of bending, tension and gravity}

Let us consider Eq. (3) in closer details. The first term in the square root corresponds to the bending of the sheet, the second one to the tension in the sheet, and the third one to gravity. Using all the measured values for $B$, and 


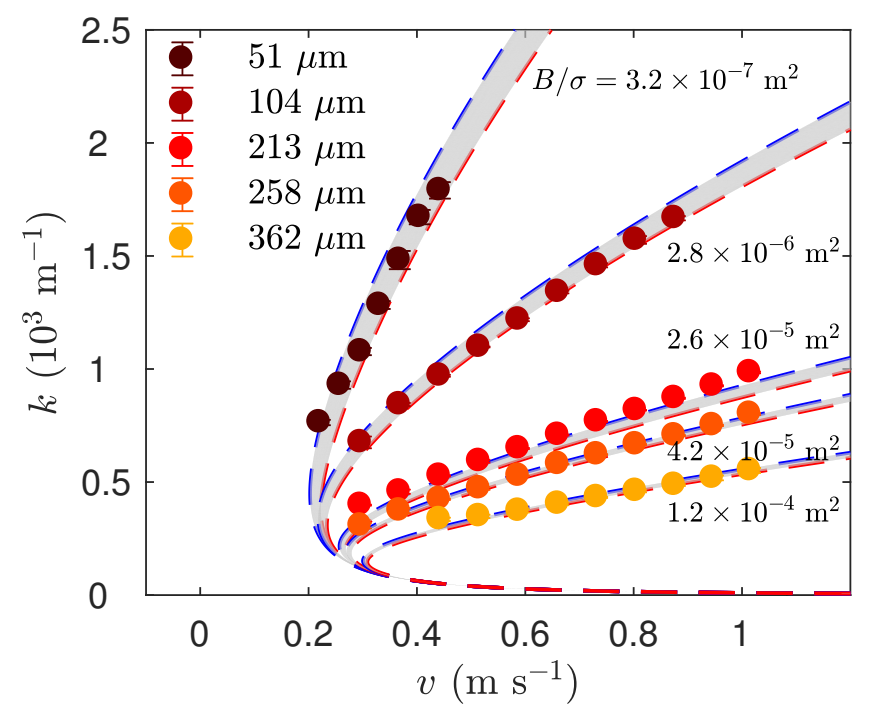

FIG. 4. Angular wavenumber $k=2 \pi / \lambda$ as a function of perturbation speed $v$, for five different sheet thicknesses $h$ as indicated in the legend. Each data point was obtained using the procedure detailed in Fig. 3 and an average over four different experimental displacement profiles. The error bars correspond to the standard deviation, which is comparable to the marker size. For each thickness $h$, the blue and red dashed lines separated by a grey region indicate the upper and lower theoretical predictions obtained from Eq. (3), using the independently-measured values of $B$ and $\sigma$ (see main text) and their uncertainties. The relative importance of bending to stretching is quantified with the ratio $B / \sigma$ and is displayed next to the corresponding curve. As the elastic sheet gets thicker (top to bottom curves), bending becomes more significant.

the value of $\sigma$, the respective contributions of those three terms, as well as their sum (dashed lines), are computed from Eq. (3) and plotted in Fig. 5. We experimentally measure angular wavenumbers ranging from 400 to $2000 \mathrm{~m}^{-1}$ (see Fig. 4). For the thinnest film $h=50 \mu \mathrm{m}$ (Fig. 5(a)), all three terms do contribute in that range. This highlights the counterintuitive role of gravity in the wavelength selection of the upstream hydroelastic waves. The elastic sheet with $h=100 \mu \mathrm{m}$ shows an interesting behaviour (Fig. 5(b)): at low angular wavenumbers $\left(k<1000 \mathrm{~m}^{-1}\right)$, all three terms are also relevant, while bending becomes predominant at larger angular wavenumbers. For the three largest thicknesses, $h=200,250$, and $350 \mu \mathrm{m}$ ((Fig. 5(c-e))), bending clearly dominates.

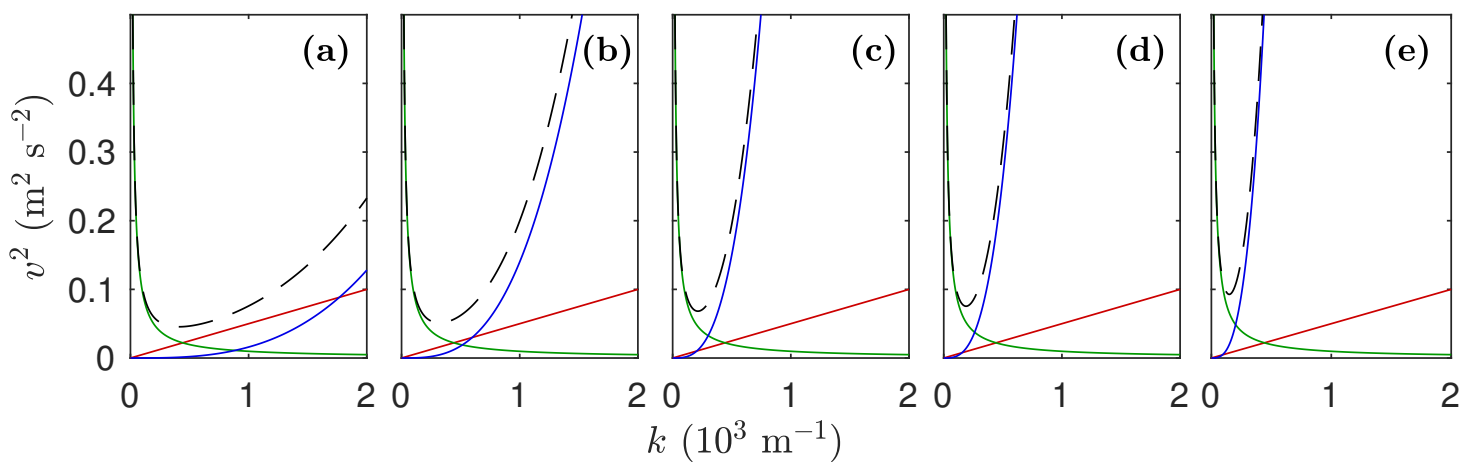

FIG. 5. Contributions of bending, tension and gravity in Eq. (3). The five panels correspond to different thicknesses (and thus bending moduli $B)$ - from left to right: $h=50,100,200,250$, and $350 \mu \mathrm{m}$. The blue line corresponds to bending $\left(B k^{3} / \rho\right)$, the red line to tension $(\sigma k / \rho)$, and the green line to gravity $(g / k)$; while the dashed line represents the sum of the three contributions. 


\section{CONCLUSION}

In this article, we have studied the hydroelastic waves formed by moving a thin elastic sheet, floating on water, past a stationary air jet. Specifically, we experimentally measured the wavelength of the wave as a function of the perturbation speed, for sheets with bending moduli varying over two orders of magnitude. For thin elastic sheets (thickness smaller than $100 \mu \mathrm{m}$ ), the tension in the sheet plays a significant role in the propagation of the waves. For thicker elastic sheets (thickness larger than $100 \mu \mathrm{m}$ ), the bending contribution becomes dominant - a regime that is particularly relevant for floating ice [20, 21, 28]. The results are found to be in excellent agreement with theoretical predictions, based on the elasticity of slender structures coupled to the hydrodynamics of inviscid incompressible flows, with no adjustable parameter. Interestingly, for thin elastic sheets, bending, tension and gravity all contribute to the hydroelastic waves - a result with practical consequences in geophysics, biophysics and civil engineering.

\section{ACKNOWLEDGMENTS}

The financial support by the Natural Science and Engineering Research Council of Canada and the Joliot Chair of ESPCI Paris is gratefully acknowledged. The authors also thank Andreas Koellnberger and Wacker Chemie AG for technical information and the donation of Elastosil ${ }^{\circledR}$ Film 2030 films, as well as to Antonin Eddi, Lucie Domino, Andreas Carlson and Yacine Amarouchene for stimulating discussions. Last but not least, they gratefully acknowledge the excellent collaboration with the McMaster Engineering Machine Shop.

\section{Appendix: Image analysis}
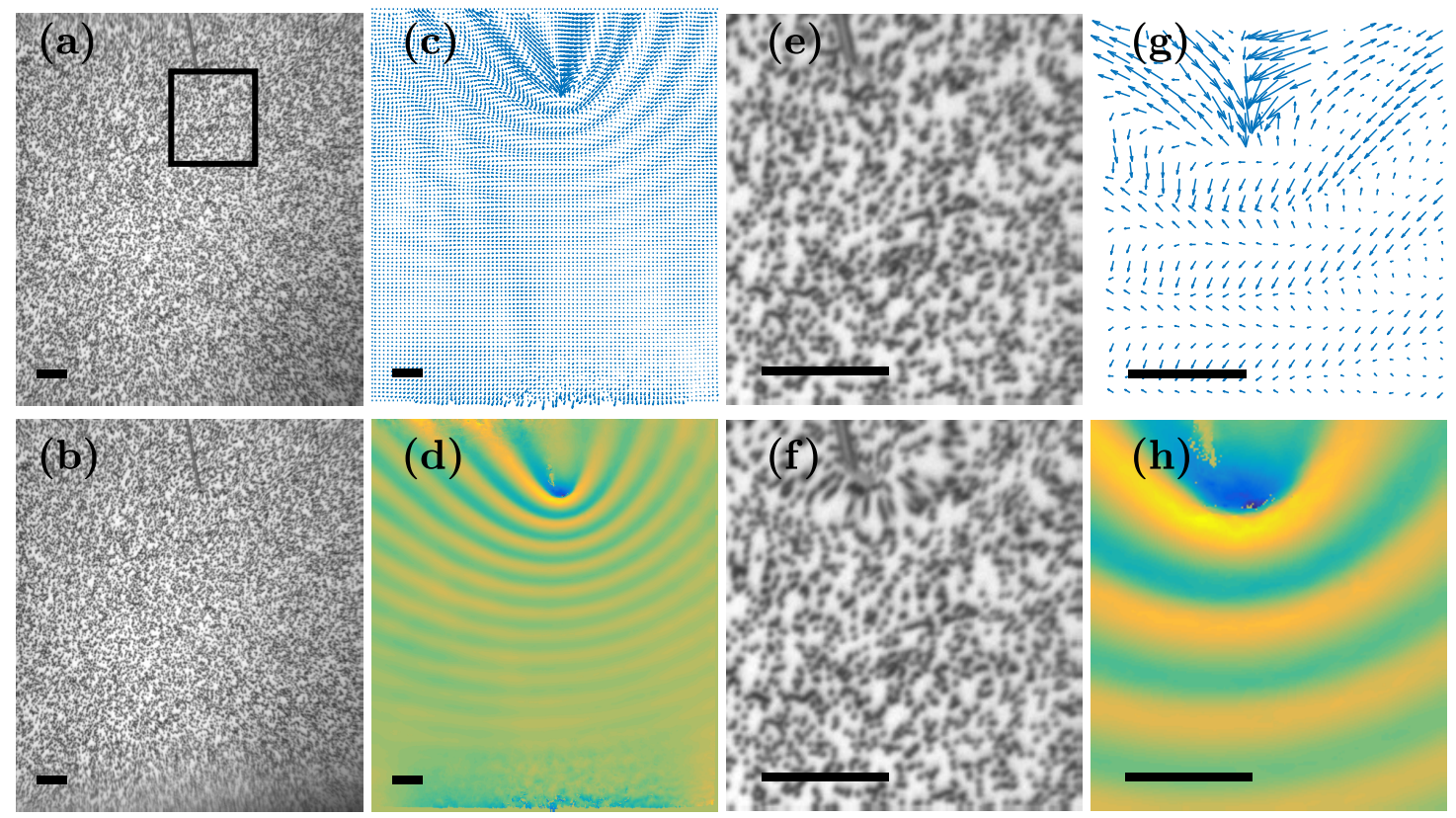

FIG. 6. (a) Raw picture of the dot pattern placed underneath the tank when no perturbation is applied (no wave propagating), called the reference image. The grey line at the top-centre of the image, just extending into the black box, is the pipette used to blow air at the surface of the elastic sheet. (b) Raw picture of the dot pattern when a perturbation is applied and hydroelastic waves are propagating (note that shear is hard to discern at this magnification - compare with (e) and (f)). (c) 2D dot-displacement data obtained with Ncorr [44] using the images shown in (a) and (b). (d) The $y$-component of the displacement field. Warm colours (green to orange) correspond to positive displacements, while cold colours (green to blue) correspond to negative ones. (e-h) zoom around the perturbation, corresponding to the black box in (a), for figures (a-d) respectively. Notice that the dot pattern (see (e)) is distorted (see (f)) as the waves propagate on the elastic sheet. This is the dot displacement quantified using Ncorr. All scale bars correspond to $1 \mathrm{~cm}$.

As described in the main text, the waves are imaged using the Schlieren method [42]. Specifically, the apparent 
displacement of the dots due to refraction at the interface is measured when hydroelastic waves are propagating on the elastic sheet. The displacement of the dots is measured by comparing the image of the dot pattern when waves are propagating (Fig. 6(b, f)) to a reference (Fig. 6(a, e)). For both images, the elastic sheet is rotating at speed $\Omega$ in the tank and the measured displacement is only a result of the waves propagating at the interface. The apparent displacement can be seen more easily when zooming closer to the perturbation (Fig. 6(f)) where the magnitude is larger. Processing these images with the open-source digital 2D image-correlation algorithm (Ncorr, Matlab) [44], Fig. $6(\mathrm{c}, \mathrm{g})$ are obtained. Finally, the strongest signal for measuring the wavelength of the waves is obtained by looking at the vertical component of the displacement vectors (Fig. $6(\mathrm{~d}, \mathrm{~h})$ ).

\section{Appendix: Wave profile analysis}

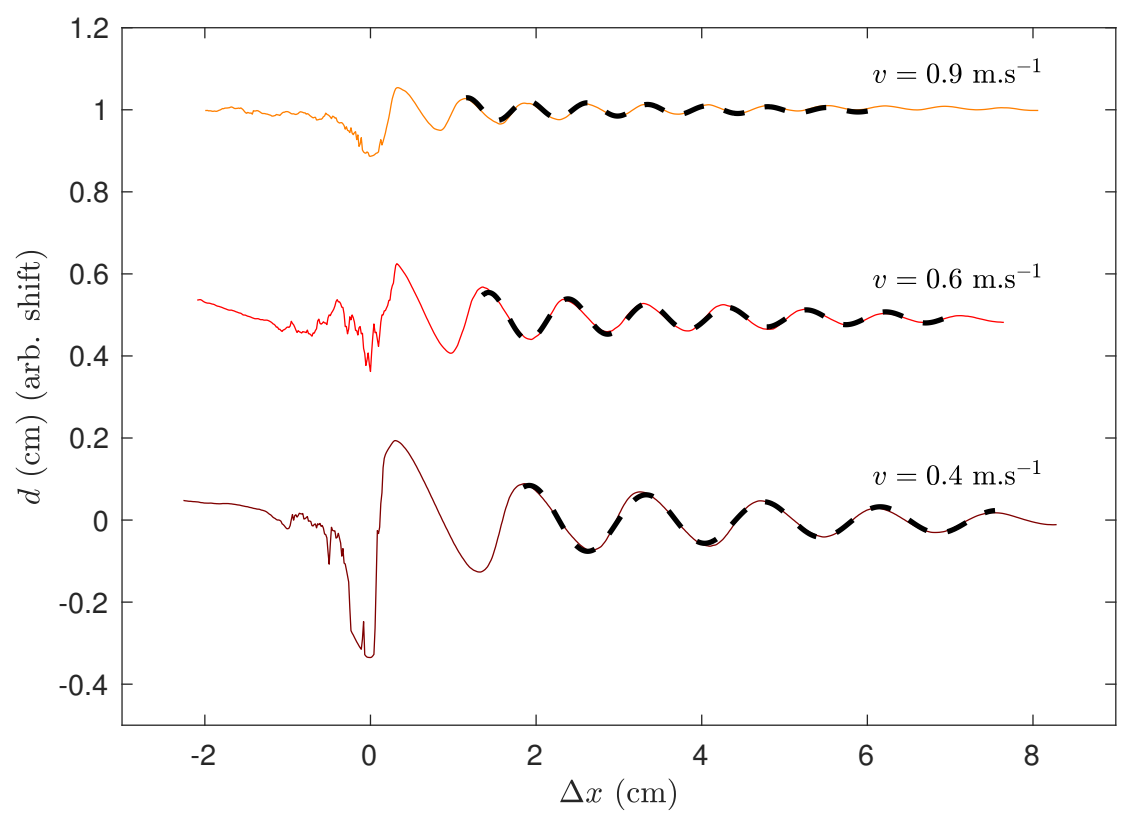

FIG. 7. $y$-component $d$ of the displacement field normal to the wave front, already shown in Fig. 3(d), for three different speeds $v=0.4,0.6$, and $0.9 \mathrm{~m} . \mathrm{s}^{-1}$. The black dashed lines show the best fit of Eq. (A.1) to the experimental profiles.

As explained in section III A., the displacement profiles normal to the wave front can be extracted and used to measure the wavelength of the hydroelastic waves. The displacement profiles can be empirically described by:

$$
d=A \exp (-\kappa x) \sin (k x+\phi)
$$

where $\kappa$ is the inverse of the characteristic length scale of the decay, $k=2 \pi / \lambda$ is the angular wavenumber, and $\phi$ is the phase of the wave. The fit is performed one wavelength away from where the perturbation is applied. Indeed, the imaging technique is optimized in order to measure precisely small deformations of the surface. Near the perturbation, the magnitude of the displacement is too large and cannot be measured precisely by the algorithm. Fitting away from the perturbation minimizes experimental artefacts as well as possible non-linearities near the perturbation. Figure 7 shows excellent agreement between this empirical description of the wave and the experimental data, indicating that the wave is dominated by a single wavelength $\lambda$. The wavelength is extracted from the fit and is consistent with a peak-to-peak measurement of the wavelength. In addition, we have performed measurements of the wavelength near and far away from the perturbation using the peak-to-peak method. Both measurements agree well within $5 \%$, the difference being most significative at lower speed $(\lambda \sim 1 \mathrm{~cm})$. Another parameter of interest is $\kappa$ which characterizes the exponential decay of the wave amplitude away from the perturbation. The profiles shown in Fig. 7 indicate that $\kappa$ increases with the speed of the perturbation, i.e. the amplitude of the wave decays faster as $v$ increases. This trend seems to hold for the different sets of experiments conducted during this study. However, a quantitative analysis of the decay constant for the different elastic sheets is beyond the scope of the current work. 


\section{Appendix: Tension in the film}

\section{Tension isotropy}
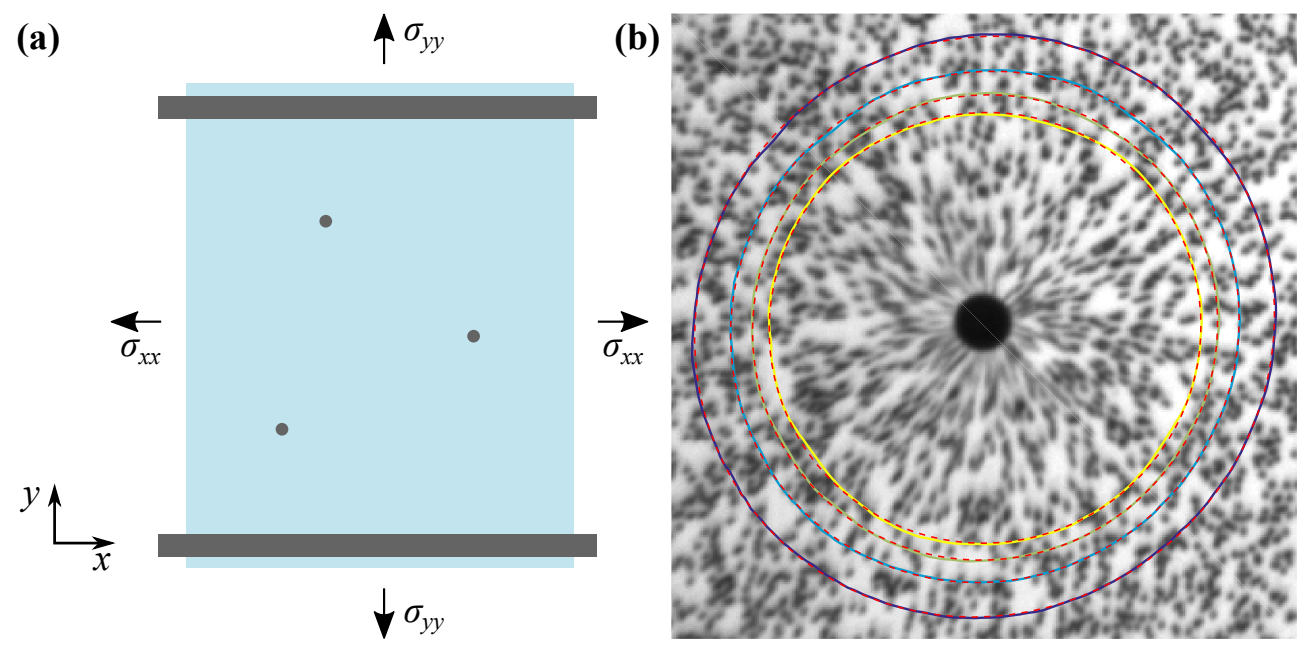

FIG. 8. (a) Top-view schematic of the elastic sheet floating on water. Plastic beams are placed along the radial edges of the elastic sheet as shown in black to ensure the sheet does not crumple. Ball bearings are dropped atop the sheet to verify that no anisotropy is introduced when placing the beams. (b) Picture of the reference dot pattern, seen through the water and the sheet, around a ball bearing. The solid lines are sample isodisplacement lines. The dashed lines are the best fits of the isodisplacement lines to ellipses. The best-fit ellipticities are found to be equal to 1 for all cases.

A schematic of the elastic sheet is shown in Fig. 8(a). The tensions along the $x$ - and $y$-axes are denoted $\sigma_{x x}$ and $\sigma_{y y}$, respectively. We place ball bearings directly on the sheet floating on water, and we image the resulting deformation using the optical Schlieren method [42]. We then calculate the magnitude of the displacement vector field, which is directly linked to the deformation of the elastic sheet. Sample iso-displacement lines are shown in Fig. 8(b). We quantify the anisotropy of the deformation by fitting the iso-displacement lines to ellipses. We define the aspect ratio $\epsilon=r_{x} / r_{y}$, where $r_{x}$ and $r_{y}$ are the radius along the $x$ and $y$ axis respectively, to quantify how circular the iso-displacement lines are. The best-fit to ellipses for the four cases shown in Fig. 8(b) are all found to be $\epsilon=1 \pm 0.02$, meaning that the iso-displacement lines are, within experimental error, equivalent to circles. Thus the tension in the sheet is isotropic. Indeed, if $\sigma_{x x}$ or $\sigma_{y y}$ was larger than the other, the deformation would be elongated along the low-tension direction, leading to an aspect ratio $\epsilon$ different from 1 .

\section{Tension measurement}

The tension $\sigma_{x x}$ along the $x$-axis is set by the water-air surface tension $\gamma$, as both the left and right edges are free (see Fig. 8(a)). Since all the experiments presented in the study are performed on sheets where the tension is isotropic, one can safely assume that $\sigma=\sigma_{y y}=\sigma_{x x}=\gamma$. The tabulated value for the pure water-air surface tension under ambient conditions is $\gamma=72 \mathrm{mN} \cdot \mathrm{m}^{-1}$, but it is extremely sensitive to contamination by all kinds of surfactants. The experiments being conducted in an open tank containing $\sim 200 \mathrm{~L}$ of water, contamination is unavoidable. Therefore, $\gamma$ was measured from two independent methods.

First, using a Wilhelmy-plate setup, $\gamma$ was found to be between 40 and $55 \mathrm{mN} \cdot \mathrm{m}^{-1}$, for water from three different sources: water from the tank after one day, tap water, and deionized water. The largest value of $\gamma$ was obtained for deionized water, and the smallest one for the water from the tank - which is consistent with tank contamination over time.

Another approach to measure $\gamma$ is to invoke the gravito-capillary waves formed at the surface of deep water by a perturbation moving at constant speed $v$. In such a case, the analogue of Eq. (3) is [7]:

$$
v=\sqrt{\frac{\gamma k}{\rho}+\frac{g}{k}} .
$$




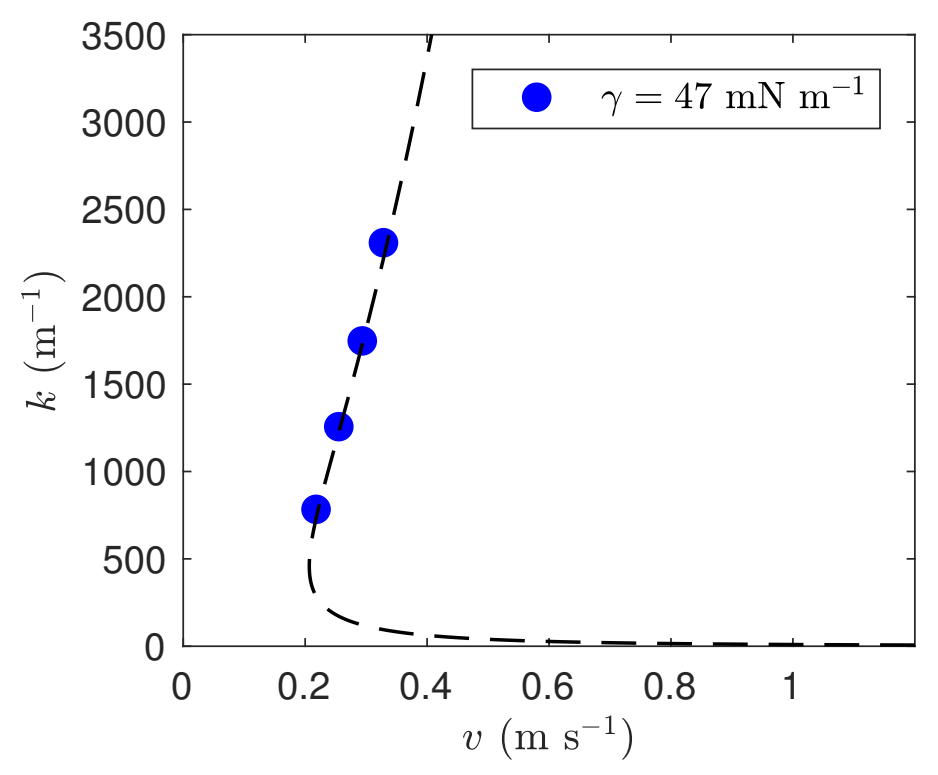

FIG. 9. Angular wavenumber $k$ as a function of perturbation speed $v$, for the gravito-capillary waves at the surface of deep water. The dashed line shows the best fit of Eq. (A.1) to the experimental data points. The water-air surface tension $\gamma=47 \mathrm{mN}^{-1}$ is obtained as the only adjustable parameter.

Therefore, $\gamma$ can be evaluated by fitting Eq. (A.1) to the experimental evolution of the angular wavenumber $k$ as a function of speed $v$ for gravito-capillary waves. In fact, as the elastic sheet only covers a small portion of the water in the tank, the hydroelastic waves are only observed once a lap when the sheet moves across the stationary perturbation at speed $v$. Otherwise, water flowing at speed $v$ is directly exposed to the perturbation, and gravito-capillary waves are instead formed at the surface. Using the Schlieren method, the wavelength $\lambda=2 \pi / k$ of the upstream gravito-capillary waves is measured as a function of speed $v$. The measurements of the wavelengths for both the hydroelastic and the gravito-capillary waves are thus performed simultaneously. Figure 9 shows the evolution of the angular wavenumber $k$ as a function the speed $v$, for the gravito-capillary waves. By fitting the experimental data to Eq. (A.1), one finds $\gamma=47 \mathrm{mN} \cdot \mathrm{m}^{-1}$.

Note that the last measurement was performed during the characterization of the hydroelastic waves on a sheet of thickness $h=50 \mu \mathrm{m}$. Similar measurements were also performed during the characterization of the hydroelastic waves on sheets with larger thicknesses $h=\{100 ; 200 ; 250\} \mu \mathrm{m}$. However, in those cases, the wavelength for the gravito-capillary waves was measurable only for the lowest speed, $v \approx 0.2 \mathrm{~m} . \mathrm{s}^{-1}$. For all those three measurements, we get $\gamma=47 \mathrm{mN} \cdot \mathrm{m}^{-1}$. Considering all the measured values from both methods, we reach the conclusion that $\gamma=50 \pm 10 \mathrm{mN} \cdot \mathrm{m}^{-1}$.

\section{Appendix: Theoretical expression for the minimal velocity and angular wavenumber}

As explained in section III B., there exists a minimal velocity $v^{*}$ below which there is no wave propagation. It is given by:

$$
v^{*}=\left(\frac{2}{27}\right)^{1 / 4} \sqrt{\frac{\sigma\left(\sqrt{12 B g \rho+\sigma^{2}}-\sigma\right)+12 B g \rho}{\rho \sqrt{B\left(\sqrt{12 B g \rho+\sigma^{2}}-\sigma\right)}}} .
$$

This minimal velocity is reached at an angular wavenumber $k^{*}$, given by:

$$
k^{*}=\sqrt{\frac{\sqrt{12 B g \rho+\sigma^{2}}-\sigma}{6 B}} .
$$


[1] Richard P. Feynman, Robert B. Leighton, and Matthew Sands, The Feynman lectures on physics, Vol. I (Basic books, 2011).

[2] Lord Kelvin, "On ship waves," Proc. Inst. Mech. Eng 3, 409-434 (1887).

[3] Marc Rabaud and Frédéric Moisy, "Ship wakes: Kelvin or mach angle?" Phys. Rev. Lett. 110, 214503 (2013).

[4] Alexandre Darmon, Michael Benzaquen, and Elie Raphaël, "Kelvin wake pattern at large froude numbers," J. Fluid Mech. Rapids 738 (2014).

[5] L D Landau and E M Lifshitz, Fluid Mechanics, 2nd ed. (Pergamon, 1987).

[6] David J Acheson, Elementary fluid dynamics (Oxford University Press, 1990).

[7] E. Raphaël and P.-G. de Gennes, "Capillary gravity waves caused by a moving disturbance: wave resistance," Phys. Rev. E 53, 3448 (1996).

[8] Frédéric Dias and Christian Kharif, "Nonlinear gravity and capillary-gravity waves," Annu. Rev. Fluid Mech. 31, 301-346 (1999).

[9] David L Hu, Brian Chan, and John WM Bush, "The hydrodynamics of water strider locomotion," Nature 424, 663-666 (2003).

[10] AD Chepelianskii, Frédéric Chevy, and Elie Raphaël, "Capillary-gravity waves generated by a slow moving object," Phys. Rev. Lett. 100, 074504 (2008).

[11] Fabien Closa, AD Chepelianskii, and Elie Raphaël, "Capillary-gravity waves generated by a sudden object motion," Phys. Fluids 22, 052107 (2010).

[12] Jonathan Voise and Jérôme Casas, "The management of fluid and wave resistances by whirligig beetles," J. Royal Soc. Interface 7, 343-352 (2010).

[13] Thomas Steinmann, Maxence Arutkin, Précillia Cochard, Elie Raphaël, Jérôme Casas, and Michael Benzaquen, "Unsteady wave pattern generation by waterstriders," Journal of Fluid Mechanics 848, 370-387 (2018).

[14] Karol Wedołowski and Marek Napiórkowski, "Dynamics of a liquid film of arbitrary thickness perturbed by a nano-object," Soft Matt. 11, 2639-2654 (2015).

[15] René Ledesma-Alonso, Michael Benzaquen, Thomas Salez, and Elie Raphaël, "Wake and wave resistance on viscous thin films," J Fluid Mech. 792, 829-849 (2016).

[16] René Ledesma-Alonso, Elie Raphaël, Thomas Salez, Philippe Tordjeman, and Dominique Legendre, "van der waals interaction between a moving nano-cylinder and a liquid thin film," Soft Matter 13, 3822-3830 (2017).

[17] L D Landau and E M Lifshitz, Theory of Elasticity, Vol. 7, 3rd ed. (Elsevier, New York, 198).

[18] Michael J Shelley and Jun Zhang, "Flapping and bending bodies interacting with fluid flows," Annu. Rev. Fluid Mech. 43, 449-465 (2011).

[19] Emmanuel Virot, Xavier Amandolese, and Pascal Hémon, "Fluttering flags: an experimental study of fluid forces," J. Fluid. Struct. 43, 385-401 (2013).

[20] Takatoshi Takizawa, "Deflection of a floating sea ice sheet induced by a moving load," Cold regions Science and Technology 11, 171-180 (1985).

[21] VA Squire, WH Robinson, PJ Langhorne, and TG Haskell, "Vehicles and aircraft on floating ice," Nature 333, 159-161 (1988).

[22] Vernon A Squire, John P Dugan, Peter Wadhams, Philip J Rottier, and Antony K Liu, "Of ocean waves and sea ice," Annu. Rev. Fluid Mech. 27, 115-168 (1995).

[23] Emilian Părău and Frederic Dias, "Nonlinear effects in the response of a floating ice plate to a moving load," J. Fluid Mech. 460, 281-305 (2002).

[24] James B Grotberg and Oliver E Jensen, "Biofluid mechanics in flexible tubes," Annu. Rev. Fluid Mech. 36 (2004).

[25] Motohiko Murai, Hiroshi Kagemoto, and Masataka Fujino, "On the hydroelastic responses of a very large floating structure in waves," Journal of marine science and technology 4, 123-153 (1999).

[26] E Watanabe, T Utsunomiya, and CM Wang, "Hydroelastic analysis of pontoon-type vlfs: a literature survey," Engineering structures 26, 245-256 (2004).

[27] Deniz Tolga Akcabay and Yin Lu Young, "Hydroelastic response and energy harvesting potential of flexible piezoelectric beams in viscous flow," Physics of Fluids 24, 054106 (2012).

[28] J. W. Davys, R. J. Hosking, and A. D. Sneyd, "Waves due to a steadily moving source on a floating ice plate," Journal of Fluid Mechanics 158, 269?287 (1985).

[29] Lucie Domino, Marc Fermigier, Emmanuel Fort, and Antonin Eddi, "Dispersion-free control of hydroelastic waves down to sub-wavelength scale," EPL (Europhysics Letters) 121, 14001 (2018).

[30] F Milinazzo, Marvin Shinbrot, and NW Evans, "A mathematical analysis of the steady response of floating ice to the uniform motion of a rectangular load," J. Fluid Mech. 287, 173-197 (1995).

[31] Emilian I Părău and Jean-Marc Vanden-Broeck, "Three-dimensional waves beneath an ice sheet due to a steadily moving pressure," Phil. Trans. R. Soc. A 369, 2973-2988 (2011).

[32] AE Hosoi and L Mahadevan, "Peeling, healing, and bursting in a lubricated elastic sheet," Physical review letters 93, 137802 (2004).

[33] Hugues Vandeparre, Sylvain Gabriele, Fabian Brau, Cyprien Gay, Kevin Kit Parker, and Pascal Damman, "Hierarchical wrinkling patterns," Soft Matter 6, 5751-5756 (2010). 
[34] John R Lister, Gunnar G Peng, and Jerome A Neufeld, "Viscous control of peeling an elastic sheet by bending and pulling," Physical review letters 111, 154501 (2013).

[35] Talal T Al-Housseiny, Ivan C Christov, and Howard A Stone, "Two-phase fluid displacement and interfacial instabilities under elastic membranes," Physical review letters 111, 034502 (2013).

[36] Andreas Carlson and L. Mahadevan, "Similarity and singularity in adhesive elastohydrodynamic touchdown," Physics of Fluids 28, 011702 (2016).

[37] Maxence Arutkin, René Ledesma-Alonso, Thomas Salez, and Elie Raphaël, "Elastohydrodynamic wake and wave resistance," J. Fluid Mech. 829, 538-550 (2017).

[38] Ousmane Kodio, Ian M. Griffiths, and Dominic Vella, "Lubricated wrinkles: Imposed constraints affect the dynamics of wrinkle coarsening," Phys. Rev. Fluids 2, 014202 (2017).

[39] Ruben Manuel Sylvester Maria Schulkes, RJ Hosking, and AD Sneyd, "Waves due to a steadily moving source on a floating ice plate. part 2," Journal of Fluid Mechanics 180, 297-318 (1987).

[40] Luc Deike, Jean-Claude Bacri, and Eric Falcon, "Nonlinear waves on the surface of a fluid covered by an elastic sheet," J. Fluid Mech. 733, 394-413 (2013).

[41] Luc Deike, Michael Berhanu, and Eric Falcon, "Experimental observation of hydroelastic three-wave interactions," Phys. Rev. Fluids 2, 064803 (2017).

[42] Frédéric Moisy, Marc Rabaud, and Kévin Salsac, "A synthetic schlieren method for the measurement of the topography of a liquid interface," Exp. Fluids 46, 1021 (2009).

[43] F. Moisy and M. Rabaud, "Free-surface synthetic schlieren (fs-ss): A tutorial,".

[44] J Blaber, B Adair, and A Antoniou, "Ncorr: open-source 2d digital image correlation matlab software," Exp. Mech. 55, 1105-1122 (2015).

[45] G. K. Batchelor, An Introduction to Fluid Dynamics (Cambridge University Press, Cambridge, England, 1967).

[46] Joseph D. Paulsen, Evan Hohlfeld, Hunter King, Jiangshui Huang, Zhanlong Qiu, Thomas P. Russell, Narayanan Menon, Dominic Vella, and Benny Davidovitch, "Curvature-induced stiffness and the spatial variation of wavelength in wrinkled sheets," Proceedings of the National Academy of Sciences 113, 1144-1149 (2016), http://www.pnas.org/content/113/5/1144.full.pdf.

[47] Dominic Vella and Benny Davidovitch, "Regimes of wrinkling in an indented floating elastic sheet," Phys. Rev. E 98, 013003 (2018). 